\title{
Gravidez Ectópica Rota com antecedente de Laqueadura Tubária: Relato de Caso
}

\section{Ectop pregnancy route with antecedent tubal ligation: Case report}

Alessandra Renata Felipe ${ }^{1}$, Djaiany Luz Viana², Denise Soares de Alcântara ${ }^{3}$

\section{RESUMO}

Introdução: Gravidez ectópica é aquela que se desenvolve fora da cavidade endometrial e é uma das complicações mais comuns do primeiro trimestre da gravidez. Objetivo: Relatar um caso de gravidez ectópica rota após Laqueadura Tubária. Metodologia: Relatou-se um caso de gravidez ectópica tubária em uma paciente de 39 anos atendida em um hospital público ao sul do Tocantins. Relato de Caso: A paciente deu entrada no serviço de emergência do hospital com quadro de dor abdominopélvica e sangramento transvaginal. Realizou exame de $\beta-\mathrm{HCG}$ e tomografia de abdome totalconfirmando diagnóstico de prenhez tubária rota. Foi submetida à laparotomia exploratória sem intercorrências, com alta hospitalar no segundo dia pós-operatório. A paciente recebeu orientação para acompanhamento médico por dois meses para tratar de anemia. Considerações Finais: É importante considerar que qualquer mulher em idade reprodutiva, com presença de dor abdominal inferior e sangramento vaginal, possa ser um caso de gravidez ectópica.

Descritores: Gravidez ectópica. Laqueadura.

\section{ABSTRACT}

Introduction: Ectopic pregnancy is one that develops outside the endometrial cavity and is one of the most common complications of the first trimester of pregnancy. Objective: To report a case of ectopic pregnancy route after Tubal Ligation. Methodology: A case of tubal ectopic pregnancy was reported in a 39-year-old patient attended at a public hospital south of Tocantins. Case Report: The patient was admitted to the emergency department of the hospital with abdominopelvic pain and transvaginal bleeding. She underwent $\beta$-HCG examination and total abdominal CT scan confirming the diagnosis of ruptured tubal pregnancy. She was submitted to exploratory laparotomy without intercurrences and was discharged on the second postoperative day. The patient received guidance for medical follow-up for two months to treat anemia. Final Considerations: It is important to consider that any woman of reproductive age with lower abdominal pain and vaginal bleeding may be a case of ectopic pregnancy.

Keywords:Ectópic pregnancy. Ligation.
2 Enfermeira graduada pela Universidade de Gurupi.

${ }^{3}$ Mestre, docente do curso de enfermagem da Universidade de Gurupi.

Endereço para

correspondência:

Universidade Gurupi, Av. Rio de Janeiro no 1585, Centro 77403-090, Gurupi - TO, Telefone: (63) 3612-7600

E-mail:

soaresdenise468@gmail.com 


\section{INTRODUÇAO}

A gravidez ectópica (GE) é a implantação do blastocisto fora da cavidade uterina, representa a primeira causa de morte materna no primeiro trimestre da gestação e a segunda causa de mortalidade materna ${ }^{1,2,3}$.

Mais de $95 \%$ de todas as GE são tubárias. As mulheres com essa patologia de localização tubária (GET), além de apresentarem um índice aumentado na taxa de infertilidade, também têm um risco aumentado para GET em gestações futuras².

Os fatores de risco associados a GE mais observados na literatura são: GE prévia, história de cirurgia tubária, tabagismo, uso de dispositivo intrauterino (DIU), história de abortos espontâneos, endometriose, laqueadura prévia, anticoncepção de emergência, infertilidade feminina, anomalias congênitas do útero, história de doença inflamatória pélvica (DIP), raça, história de infecções sexualmente transmissíveis (DST's) e múltiplos parceiros sexuais ${ }^{5}$.

Diante da diversidade de fatores de risco e dificuldade de se estabelecer esses riscos em diferentes populações, o diagnóstico de GE segundo a literatura ${ }^{6}$ tem se embasado especialmente na associação da ultrassonografia transvaginal e dosagem do $\beta$ - HCG gonadotrofina coriônica humana como método de diagnóstico desta enfermidade 6 .

$\mathrm{Na}$ abordagem diagnóstica há de se considerar que a gravidez ectópica tem se comportado de maneira diferenciada nas últimas décadas, o que muda de maneira drástica seu diagnóstico, o qual antes era feito baseado na presença de choque hemorrágico por ruptura da GE, agora já tem sido feito por um quadro de sintomas não específicos, que ocorrem antes da ruptura ${ }^{6}$.

Apesar do diagnóstico precoceter diminuído a mortalidade por GE ao longo das últimas décadas, nos Estados Unidos ainda são responsáveis por $9 \%$ das hemorragias maternas no primeiro trimestre de gravidez e no Brasil a mortalidade está inserida entre as causas hemorrágicas ${ }^{7}$.

Casos de gravidez ectópica diagnosticados antes da ruptura tubária, podem ser tratados por condutas conservadoras, como a laparoscopia com salpingostomia, o tratamento medicamentoso com Metrotexato (MTX) e a conduta expectante ${ }^{6}$.

A cirurgia laparoscópica é o tratamento de primeira escolha nos casos de GE, no entanto quando a paciente tem intenção de engravidar surgem controvérsias entre a cirurgia radical (salpingectomia) e a cirurgia conservadora (salpingostomia) ${ }^{8}$. 
Por serem incomum as descrições de gravidez ectópica após laqueadura, objetivase neste artigo atentar profissionais de saúde para esta suspeita diagnóstica, por vezes pouco lembrada no cotidiano.

\section{MATERIAIS E METODOS}

Os dados contidos neste caso clínico foram obtidos através de entrevista, com uma das autoras do presente relato e exames da mesma, após autorização prévia desta. Utilizou-se registro fotográfico dos métodos diagnósticos, aos quais a paciente foi submetida e revisão de literatura.

\section{DESENVOLVIMENTO}

Paciente de 39 anos, branca, Gesta 3, Para 2, com história negativa para Doença Inflamatória Pélvica (DIP), submetida a laqueadura há 11 anos atrás, procurou a urgência de um hospital particular relatando dor abdominopélvica e sangramento transvaginal com perda de "uma pele negra, semelhante a uma massa". Esclareceu que a dor havia se iniciado há dois dias atrás, onde a mesma procurou o serviço de saúde em sua cidade de domicílio (Figueirópolis-TO), sendo medicada com analgésico e encaminhada de volta para casa. Relata que como a dor se intensificou muito, decidiu buscar um serviço particular. Foi hospitalizada imediatamente, onde permaneceu por dois dias sendo submetida a vários exames laboratoriais, ultrassonografia de abdômen total, pélvica e transvaginal, Radiografia do tórax e abdômen. Foi medicada com analgésicos para o alívio da dor. Os achados na ultrassonografia de abdômen total revelaram pequena coleção líquida em retrocavidade dos epiplons, pelve e fundo de saco, o que resultou na hipótese diagnóstica de líquido livre em abdômen, em maior quantidade a esquerda, cauda de pâncreas não visualizada, restante do abdome sem alterações (como consta Fig. 1).

Diante dos resultados da Ultrassonografia o médico que conduzia o caso, informou a paciente que ficaria mais alguns dias hospitalizada afim de esclarecer o diagnóstico. No terceiro dia de internação, por falta de recursos financeiros, a paciente solicitou e foi transferida para um hospital público. Quando admitida na emergência do Hospital Público apresentava náuseas sem vômitos, dor em região epigástrica e baixo ventre, limitando a deambulação. 
Ao exame físico, abdômen sem sinais de irritação peritoneal, Blumberg negativo. Sangramento vaginal persistia em pequena quantidade. Coletou amostra de sangue para exame de $\beta$-HCG e foi encaminhada para tomografia de abdômen total, sendo diagnosticada com gravidez ectópica. A partir do diagnóstico, a paciente foi imediatamente encaminhada para o centro cirúrgico sendo submetida à laparotomia exploradora, encontrado tuba uterina rota a esquerda com material embrionário em seu interior. Foi realizada salpingectomia à esquerda, limpeza e aspiração da cavidade abdominal que continha sangue em pouca quantidade. Não houve intercorrências cirúrgicas, não foi necessária reposição de hemoderivados. O material cirúrgico foi enviado para exame histopatológico, confirmando prenhez tubária rota. A paciente recebeu alta no segundo dia pós-operatório, sendo necessário acompanhamento por dois meses, devido presença de anemia a qual foi tratada com Sulfato Ferroso.

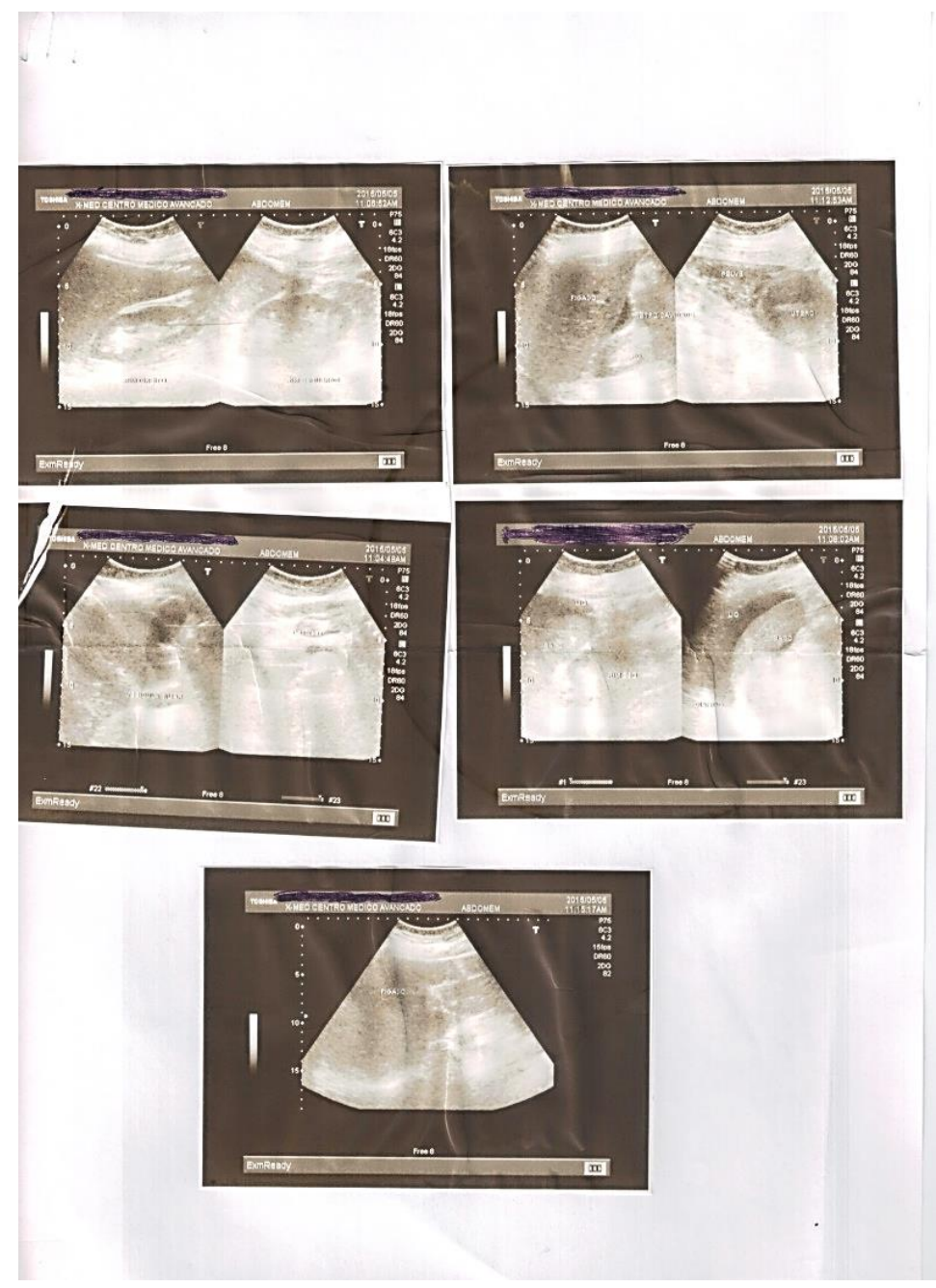

Figura 1 (Ultrassonografia) 
A prenhez ectópica é definida como a implantação do óvulo fertilizado em qualquer lugar diferente de sua localização endometrial. É uma séria emergência ginecológica considerada como entidade hemorrágica grave. Vários são os fatores de risco associados a GE. A cirurgia tubária prévia é considerada um importante fator de risco. No caso em questão, o antecedente de laqueadura se apresenta como fator de risco evidente, considerando que a paciente havia sido submetida a esta intervenção há 11 anos atrás.

É primordial avaliar todas as mulheres que apresentem queixas de dor importante e sangramento, após um longo período de amenorreia, pois tem grande probabilidade para diagnóstico de $\mathrm{GE}^{9}$.

A tríade clássica para uma GE é dor abdominal, ausência de menstruação e hemorragia vaginal embora só está presente em cerca de $50 \%$ das mulheres com esta condição ${ }^{9}$.

A GE pode ter poucos ou vários sintomas, porém, em alguns casos, pode ser assintomática; condição de evolução insidiosa, faz com que poucas mulheres busquem tratamento, acarretando muitos danos nas trompas de falópio. Esses danos podem ser parciais ou totais, e ter como sequelas a esterilidade definitiva ou dor pélvica crônica ${ }^{10}$.

As manifestações clínicas apresentadas pela paciente direcionavam o diagnóstico como um caso GE, porém sua confirmação foi tardia, o fato da paciente ser laqueada mascarou o diagnóstico. Nos últimos anos, a melhora nos métodos diagnósticos, especialmente a associação das ultrassonografias transvaginais com a dosagem da subunidade $\beta$-HCG da gonadotrofina coriônica humana, tem proporcionado o diagnóstico de forma satisfatória desta condição ${ }^{6}$.

No presente relato, quando a gravidez ectópica foi diagnosticada, a tuba já se encontrava rota. A gravidez ectópica rota possui alta incidência em nosso meio, justificada pelo fato do diagnóstico não ser feito precocemente em razão da procura tardia das pacientes aos serviços de urgência, ou por ausência de recursos laboratoriais nos serviços públicos, ou mesmo quando estes estão disponíveis, mas os médicos não os utilizam de maneira adequada ${ }^{5,6}$

O diagnóstico precoce possibilita a adoção de medidas terapêuticas tanto clínicas (Metotrexato) quanto cirúrgicas (cirurgia laparoscópica) conservando tanto a vida como a fertilidade das pacientes ${ }^{11}$.

O diagnóstico impreciso ou tardio pode comprometer a fertilidade da mulher ou até mesmo levá-la a um prognóstico ruim. O diagnóstico tardio delonga o tratamento cirúrgico 
predispondo a perda da trompa ou do anexo, eleva o tempo de internação e aumenta a chance de complicações como anemia, acarretando hemotransfusões ${ }^{13}$.

A gravidez ectópica não deve ser desconsiderada em mulheres submetidas a laqueadura tubária, especialmente se dois ou mais anos se passaram desde a esterilização ${ }^{14}$.

O prognóstico materno depende de fatores como diagnóstico precoce, a necessidade de transfusão sanguínea ${ }^{12}$.

O desfecho deste caso, embora a paciente tenha sido submetida a cirurgia de urgência e posteriormente recebido tratamento para anemia, foi bem-sucedido. Mas não exime o alerta para o aprimoramento de estratégias de diagnóstico precoce de GE a fim de melhorar a assistência de atendimento dessas mulheres, e evitar danos que causam a infertilidade.

\section{CONSIDERAÇOES FINAIS}

Concluímos que mulheres em idade reprodutiva com sinais e sintomas de dor em abdome inferior ou sangramento vaginal, deve ter suspeita de diagnóstico de GE. O diagnóstico precoce pode evitar complicações e até a morte. No caso em questão, o diagnóstico foi prejudicado pelo fato da paciente ser laqueada, o que afastou a possibilidade de uma hipótese de gravidez. Os médicos devem estar atentos de que gravidezes e podem ocorrer mesmo em mulheres laqueadas.

\section{REFERENCIAS}

1. Andersen, ANO, et al. Maternal age and fetal loss: Poplation Based Register Linkage study. 2000; 17081712.

2. Shao R. Understanding the mechanisms of human tubal ectopic pregnancies: Newevidence from knockout mouse models. Human reproduction, Gothenburg. 2009; 25 (3): 584-587.

3. Sivalingam VN. et al. Diagnosis and management of ectopic pregnancy. UKPMC Funders Group, Edinburgh. 2011; 231-240.

4. Fernandez $\mathrm{H}$, Gervaise A. Ectopic pregnancies after infertility treatment: Modern diagnosis and therapeutic strategy. Hum Reprod Update. 2004; 10 (6): 503-513.

5. Lin EP. et al. Diagnostic clues to Ectopic Pregnancy, Radio Graphics. 2008;(28): 1661-1671.

6. Soares RC, Elito Junior J, Camano L. Relação entre a espessura endometrial e os níveis do beta-hCG com resposta ao tratamento da Gravidez Ectópica com Metotrexato. RBGO. 2004; (26): 471-475. 
7. Fernandes, MAS. et al. Prevalência de Gestação Ectópica de Tratamento Cirúrgico em Hospital Público de 1995-2000. Rev. Assoc. Med. Bras. 2004; 50 (4): 413-416.

8. Silva PD, Schaper AM, Rooney B. Reproductive Outcome After 143 Paparoscopic Procedures for Ectopic Pregnancy. Obstet Gynecol. 1993; 81: 710-715.

9. Michael Sheele J, Sheele JM, Rachel B, Counselman FL. A Ruptured Ectopic PregnancyPresent In with a Negative Urine Pregnancy Test. Case Rep EmergMed 2016; 1-3.

10.Marques, CAS, Menezes, MLB. Infecção Genital por Chlamydia Trachomatis e Esterilidade. Dst - J. Brás. Doenças Sex. Transm, 2005; 66-70.

11.Elito Junior J, Uchiyama, MN, Camano I. Gravidez Ectópica Cervical com Embrião Vivo: Relato de quatro caso. Rev. Bras. Ginecol. Obstet.,1999; 347-350.

12.Pereira PP. Qual é a Melhor Conduta na Prenhez Ectópica? Ver. Assoc. Med. Br; 2001; 180.

13.Chaves Neto H. Obstetrícia Básica. São Paulo: Atheneu, 2005.

14.Shan JP, Parulekar SV, Hinduísia IN.Gravidez Ectópica após esterilização tubária.J. Postgrad Med. 1991 Jan; 37 (1): 17-20. 